\title{
sCD36 sérico y variables cardiometabólicas en dos poblaciones peruanas: Carhuamayo (4100 msnm) y Mala (30 msnm)
}

\author{
Elizabeth Carranza Alva ${ }^{1, a}$, Carmen Peña Suasnábar 2,a , Yadira Fernández Jeri ${ }^{2, a}$, Alejandro Florentini Carranza ${ }^{3, a}$
}

\section{RESUMEN}

Objetivo: Determinar los niveles séricos de sCD36, molécula relacionada al metabolismo lipídico, en poblaciones de la altura y del nivel del mar, y establecer la asociación de este parámetro con factores de riesgo cardiometabólico.

Materiales y métodos: Participaron 45 personas de Carhuamayo (4100 msnm) y 40 personas de Mala (30 msnm). Se midió el peso, talla y presión arterial. Se determinó la hemoglobina en sangre total, y la glucosa, perfil lipídico y sCD36 en suero.

Resultados: Se encontró en la población de Carhuamayo niveles de hemoglobina significativamente mayores, mientras que el peso, IMC y nivel de glucosa fueron significativamente menores que en Mala. No hubo diferencia significativa entre los niveles séricos de SCD36 de ambas poblaciones. Se observó una diferencia significativa entre los valores medios de sCD36 según el IMC, y una correlación positiva significativa entre sCD36 y el peso e IMC.

Conclusiones: El nivel sérico observado de sCD36 es independiente de la altitud y puede ser considerado como marcador potencial de síndrome metabólico.

Palabras clave: sCD36; factor de riesgo cardiometabólico; obesidad; altitud (Fuente: DeCS BIREME).

\section{Serum levels of sCD36 and cardiometabolic variables in two Peruvian towns: Carhuamayo (4100 masl) and Mala (30 masl)}

\section{ABSTRACT}

Objectives: To determine the serum levels of sCD36, a lipid metabolism-related molecule, in high-altitude and sea-level populations, and to establish the association of this parameter with cardiometabolic risk factors.

Materials and methods: The study population consisted of 45 people from Carhuamayo (4100 masl) and 40 people from Mala (30 masl). Weight, height and blood pressure were measured. Hemoglobin was determined in whole blood, and glucose, lipid profile and sCD36 in serum.

Results: It has been found that hemoglobin levels in the population of Carhuamayo were significantly higher, while weight, BMI and glucose level were significantly lower than those in the population of Mala. There was no significant difference between serum levels of SCD36 in both populations. A significant difference was observed between sCD36 mean serum levels of both populations based on the BMI, and a significant positive correlation between sCD36 and the weight and BMI.

Conclusions: The observed SCD36 serum level is not related to the altitude and can be considered as a potential marker of metabolic syndrome.

Keywords: sCD36; cardiometabolic risk factor; obesity; altitude (Source: MeSH NLM).

1. Instituto Nacional de Biología Andina, Facultad de Medicina.

2. Facultad de Farmacia y Bioquímica.

3. Facultad de Ciencias Biológicas.

a. Universidad Nacional Mayor de San Marcos. Lima, Perú. 


\section{INTRODUCCIÓN}

El receptor CD36 pertenece a la familia de receptores "scavenger" de tipo B y es una proteína glicosilada de 88 $\mathrm{kDa}$, multifuncional, se expresa en una variedad de células y tejidos, tales como células epiteliales, adipocitos, hepatocitos, monocitos y macrófagos, plaquetas, células del músculo esquelético y cardíaco, etc. ${ }^{(1,2)}$. Está codificada por el gen humano $\mathrm{Cd} 36$, que se encuentra en el cromosoma 7 (7q11.2) y consta de 15 exones. La proteína CD36 se compone de una sola cadena peptídica de 472 aminoácidos y está organizado en dos dominios transmembrana: dos dominios citoplasmáticos muy cortos y un gran dominio extracelular glicosilado ${ }^{(3)}$.

El receptor CD36 está involucrado en el metabolismo de los lípidos, tiene un papel importante en la internalización de los ácidos grasos libres hacia el interior de la célula, así en el caso de los miocitos, la r-oxidación de los ácidos grasos en la mitocondria juega un papel primordial en la producción de energía vital, y en los adipocitos sirve para la acumulación y reserva de lípidos ${ }^{(4)}$. Como receptor depurador ("scavenger") tiene la capacidad de unirse con ligandos tales como productos finales de glicación avanzada (AGE), lipoproteínas de baja densidad oxidada (LDL-Ox), trombospondina y neutrófilos ${ }^{(5)}$.

El CD36 está implicado en numerosas condiciones patológicas asociadas con la desregulación metabólica, tales como la obesidad, la resistencia a la insulina, diabetes, nefropatía diabética y aterosclerosis ${ }^{(6)}$. La formación de células espumosas es el paso inicial de la aterosclerosis. El proceso aterogénico comienza con la transmigración de los monocitos de sangre que circula en la íntima arterial, donde se convierten en macrófagos ${ }^{(7)}$ los cuales internalizan LDLox y LDL-glicada a través de su receptor CD36 ${ }^{(8)}$. En 1993 , Endemann et al $^{(8)}$ identificaron a la molécula CD36 como un potencial receptor de LDL-ox. Se encontró que CD36 puede reconocer LDL que han sido expuestas a condiciones "mínimamente" oxidantes, y que fueron modificadas, por ejemplo, por el sistema "peróxinitrito-mieloperoxidasaH2O2" de las células fagociticas ${ }^{(9)}$. La interacción entre CD36 y LDL-ox también induce la secreción de citoquinas que activan la proliferación y migración de las células hacia la íntima arterial ${ }^{(10)}$, y la inflamación arterial provocada por las células espumosas puede producir con el tiempo un estrechamiento de la luz arterial ${ }^{(10)}$.

Aunque el receptor CD36 es conocida como una glicoproteína de membrana, se ha identificado en el plasma humano una forma circulante de CD36, y se le ha denominado CD36 soluble $(\mathrm{sCD} 36)^{(11)}$. Se ha reportado un nivel elevado de sCD36 plasmático en diferentes estados patológicos como lesión hepática ${ }^{(12)}$, obesidad ${ }^{(11,13)}$ resistencia a la insulina ${ }^{(11,14)}$, aterosclerosis, inestabilidad de la placa, activación de monocitos e inflamación ${ }^{(15,16)}$, etc.

Estudios recientes indican que la glicación de las partículas de LDL aumenta la expresión del receptor CD36, con más fuerza que su oxidación. Parece que en la diabetes, la glicación de las partículas de LDL son las que inician la formación de células espumosas ${ }^{(17,18)}$. Algunos investigadores han encontrado que los monocitos en sujetos con hiperglucemia tienen aumentada la expresión del receptor CD36 en comparación con aquellos sujetos con concentración normal de glucosa ${ }^{(19)}$. Además, se ha encontrado que pacientes con mutaciones en el gen que codifica CD36 son más propensos a tener diabetes tipo 2 (20) y que la expresión del receptor CD36 en macrófagos en pacientes con diabetes es directamente proporcional al nivel sanguíneo de glucosa ${ }^{(21)}$. También se ha encontrado una expresión incrementada del receptor CD36 en macrófagos en obesidad determinada genéticamente, la cual está asociada a resistencia a insulina. Esta es probablemente una consecuencia de alteraciones de la señalización de la insulina en estas células ${ }^{(22,23)}$. En este sentido, se ha informado un incremento de hasta 4 veces de sCD36 en plasma en los pacientes DT 2 obesos, en comparación con sujetos delgados sanos ${ }^{(11)}$.

Se conoce que los habitantes de las grandes alturas presentan menor incidencia de enfermedades coronarias que los de nivel del mar ${ }^{(24)}$; se ha encontrado menor tasa de colesterol total, menor prevalencia de hipertensión arterial y obesidad, y el diabético de altura está en menor riesgo de presentar enfermedad cerebro-vascular que el diabético de nivel del mar, en razón de las menores alteraciones lipídicas que presenta ${ }^{(25)}$.

El presente estudio tiene como objetivo, determinar los niveles séricos de sCD36, su distribución y su asociación con varios factores de riesgo relacionados con enfermedades cardiometabólicas en una población de nivel del mar y otra de la altura.

\section{MATERIALES Y MÉTODOS}

\section{Sujetos de experiencia}

El presente es un estudio transversal en el que se incluyeron a 85 personas adultas, $45(10 \mathrm{H} / 35 \mathrm{M})$ nativas de la ciudad de Carhuamayo (Región Junín, $4100 \mathrm{msnm}$ ) y $40(15 \mathrm{H} / 25 \mathrm{M})$, que viven en la ciudad de Mala (Region Lima, $30 \mathrm{msnm})$. Ninguno de ellos padecía de diabetes mellitus ni enfermedad cardiovascular. Los sujetos de estudio se presentaron a primera hora de la mañana para la toma de muestra respectiva, en condiciones de ayuno absoluto (12 horas previas) y sin haber realizado actividad física. A las personas participantes se les informó el motivo de la investigación y se les hizo firmar una carta de consentimiento informado. Los datos proporcionados se consideran anónimos y confidenciales.

\section{Recolección de las muestras}

Las muestras sanguíneas, $10 \mathrm{~mL}$, fueron obtenidas mediante punción venosa del antebrazo previa asepsia, y se las distribuyó en viales con anticoagulante EDTA para la determinación de hemoglobina y en tubos de ensayo sin anticoagulante, limpios y esterilizados. La sangre extraída se dejó reposar por 30 minutos para la coagulación y luego se centrifugó por 5 minutos a 4000 
rpm para separar el suero. Se colocaron los sueros entre 2 y $5^{\circ} \mathrm{C}$ en un recipiente hermético, para ser transportados al laboratorio de Bioquímica del Instituto y almacenados a $-20^{\circ} \mathrm{C}$ hasta el momento del análisis.

\section{Mediciones}

La medición de la presión arterial la efectuó un profesional de la salud en el brazo izquierdo, con la persona sentada; se pesaron y tallaron los individuos sin calzado y con ropa ligera.

\section{Determinaciones}

Se determinaron: la concentración de hemoglobina $(\mathrm{Hb})$ en sangre total y los niveles séricos de glucosa, colesterol total, HDL-col y triglicéridos por métodos enzimáticocolorimétricos convencionales usando kits comerciales. La concentración de LDL-colesterol (LDL-col) se determinó aplicando la fórmula de Friedewald ${ }^{(26)}$. El nivel sérico de sCD36 se determinó por el método de ELISA utilizando un kit comercial marca MyBioSource, INC. (USA). De acuerdo a normas internacionales, el índice de masa corporal (IMC, peso en $\mathrm{kg}$, dividido entre el cuadrado de la estatura, en metros) entre $18,5-24,9 \mathrm{~kg} / \mathrm{m} 2$ fue considerado como peso normal; entre $25-29,9 \mathrm{~kg} / \mathrm{m} 2$ como sobrepeso y el IMC $\geq 30$ $\mathrm{kg} / \mathrm{m} 2$, como criterio de obesidad ${ }^{(26)}$.

\section{Análisis estadístico}

Los resultados fueron expresados en valores medios y desviación estándar. Se compararon las medias según la prueba $\mathrm{t}$ de Student para dos muestras independientes, la comparación múltiple se efectuó mediante análisis de varianza (ANOVA) y la subsiguiente prueba de Tamhane y se evaluó el grado de asociación mediante el coeficiente de correlación de Pearson. Se consideró significativo todo resultado cuyo valor asociado de $p$ fuera $<0,05$.

\section{Resultados}

La tabla 1, muestra las características de la población participante. La media de edad de la población de Carhuamayo fue 46,9 $\pm 10,5$ años y de la población de Mala fue 47,9 $\pm 16,2$ años, sin diferencia significativa ( $p$ $=0,706)$. No se encontraron diferencias significativas en presión sistólica y presión diastólica. Los promedios de los pesos de ambas poblaciones, $62,7 \pm 8,9 \mathrm{Kg}$ en Carhuamayo y $72,9 \pm 13,9 \mathrm{Kg}$ en la población de Mala, presentan una alta diferencia estadística $(p=0,000)$. El IMC sigue la misma tendencia por lo que se presenta una alta diferencia estadística $(p=0,000)$ entre ambas poblaciones. El sobrepeso de la población total fue de $49,4 \%$ y la obesidad fue de $21,2 \%$.

Tabla 1. Características de la población de estudio altitud

$\begin{array}{lccc} & \begin{array}{c}\text { Carhuamayo } \\ \text { (Media } \pm \text { DE) }\end{array} & \begin{array}{c}\text { Mala } \\ \text { (Media } \pm \text { DE) }\end{array} & \text { p } \\ \text { n } & 45 & 40 & 0,706 \\ \text { Edad, años } & 46,9 \pm 10,5 & 72,9 \pm 16,2 & 0,000^{*} \\ \text { Peso, kg } & 62,7 \pm 8,9 & 1,57 \pm 0,1 & 0,332 \\ \text { Talla, } \mathbf{m} & 1,56 \pm 0,1 & 29,2 \pm 4,5 & 0,000^{*} \\ \text { IMC, kg/m2 } & 25,8 \pm 3,3 & 95,7 \pm 14,3 & 0.005^{*} \\ \text { Cintura, cm } & 88,6 \pm 7,3 & 12,5 \pm 3,0 & 0,417 \\ \text { Presión sistólica, } \mathbf{m m H g} & 12,0 \pm 1,7 & 7,6 \pm 1,6 & 0,080\end{array}$

*Estadísticamente significativo (t de Student para medias)

Cuando estas variables se comparan según género y altitud, se observa que los hombres de Carhuamayo presentan menor peso $(\mathrm{kg})$ que sus similares de Mala $(70,1 \pm 4,8$ vs $80,5 \pm 10,3$; $p=0,08)$; menor IMC $(26,1 \pm 1,7$ vs $29,3 \pm 3,0 ; p=0,07)$, menor circunferencia de cintura $(95,1 \pm 2,4$ vs $103,3 \pm 7,6$; $p=0,003)$. Las mujeres de Carhuamayo también presentan menor peso que las mujeres de Mala $(60,6 \pm 8,7$ vs $68,3 \pm$ $13,3 ; p=0,01)$; $y$ menor IMC $(25,7 \pm 3,6$ vs $29,1 \pm 5,2 ; p=$ $0,003)$. No se encontró significación estadística en relación a la circunferencia de cintura. Tampoco se encontraron diferencias significativas según género por altitud en edad, talla, presión sistólica y presión diastólica.
Los resultados de hemoglobina y demás parámetros bioquímicos se presentan en la Tabla 2. Los niveles de hemoglobina en la población de Carhuamayo $(17,4$ $\pm 1,9 \mathrm{~g} / \mathrm{dL})$ y en la población de Mala $(13,2 \pm 1,2 \mathrm{~g} /$ $\mathrm{dL})$ difieren estadísticamente, $(\mathrm{p}=0,000)$, igual diferencia significativa se encontró entre los niveles de glucosa sérica de ambas poblaciones (73,2 \pm 12,6 $\mathrm{mg} / \mathrm{dL}$ vs $101,9 \pm 25,1 ; \mathrm{p}=0,000)$. No se encontraron diferencias significativas en cuanto a niveles séricos de los parámetros lipídicos estudiados. 
Tabla 2. Características de la población de estudio altitud

\begin{tabular}{lrrr} 
& $\begin{array}{c}\text { Carhuamayo } \\
\text { (Media } \pm \text { DE) }\end{array}$ & \multicolumn{1}{c}{$\begin{array}{c}\text { Mala } \\
\text { (Media } \pm \text { DE) }\end{array}$} & p \\
n & 45 & $\mathbf{4 0}$ & $0,000^{*}$ \\
Hemoglobina, g/dL & $17,4 \pm 1,9$ & $13,2 \pm 1,15$ & $0,000^{*}$ \\
Glucosa, mg/dL & $73,2 \pm 12,6$ & $212,0 \pm 34,2$ & 0,163 \\
Colesterol, mg/dL & $199,5 \pm 46,7$ & $54,5 \pm 13,8$ & 0,733 \\
HDL-colesterol, mg/dL & $55,8 \pm 20,2$ & $118,9 \pm 30,9$ & 0,296 \\
LDL-colesterol, mg/dL & $111,1 \pm 37,4$ & $193,1 \pm 128,3$ & 0,223
\end{tabular}

*Estadísticamente significativo (t de Student para medias)

Los niveles de hemoglobina fueron mayores $(p=0,000)$ tanto en hombres como mujeres cuando se les compara según la altitud, $17,9 \pm 2,7$ vs $13,9 \pm 0,9$ y $16,6 \pm 1,1$ vs $13,0 \pm 0,8$, para hombres y mujeres respectivamente. Los niveles de glucosa tanto de hombres como de mujeres de Carhuamayo fueron menores $(p=0,000)$ que sus similares de Mala $(69,2 \pm 8,5$ vs $102,2 \pm 10,6$ y $75,7 \pm 12,1$ vs 101,7 $\pm 31,0$ respectivamente. No se encontraron diferencias significativas según género por altitud en el perfil lipídico y niveles de sCD36.

En la Tabla 3 se presentan la distribución de los niveles séricos de sCD36 en la población total, y su respectivo histograma.

Tabla 3. Niveles séricos de sCD36 en la población estudiada $(n=85)$

$\begin{array}{cccccc}\text { Variable } & \text { Media } \pm \mathrm{DE} & \text { V.E. } & \text { Asimetría } & \text { Curtosis }^{\text {Normal }^{*}} \\ \mathbf{s C D} 36(\mathrm{ng} / \mathrm{mL}) & 4,52 \pm 0,89 & 2,26-6,79 & 0,52 & 0,473 & 0,017\end{array}$

*Desviación de la normalidad, prueba de Kolmogorov-Smirnov-Lilliefors

Los datos de sCD36 presentan asimetría positiva no significativa, apuntamiento no significativo, esto es, datos no concentrados alrededor de la media. La prueba de Kolmogorov-Smirnov con corrección de Lilliefors da como resultado procedencia de una población no normal para los datos. Esto se comprueba observando el histograma correspondiente.

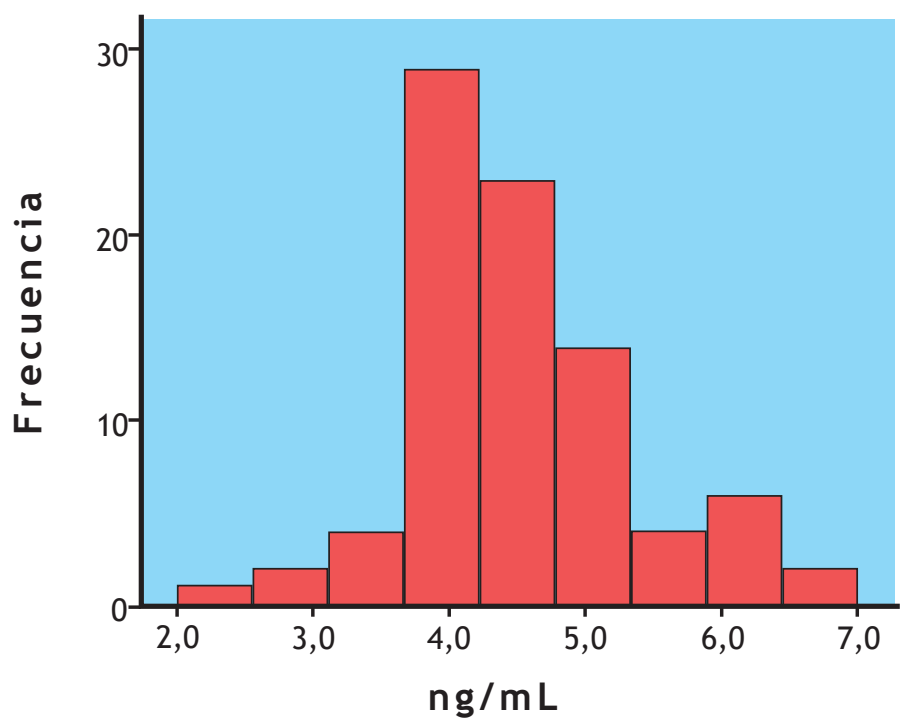

Figura 1. Distribución de frecuencias de SCD36 sérico 
La comparación de los valores medios de sCD36: 4,8 \pm 1,8 ng/mL para la población de Mala y 4,4 \pm 0,8 ng/mL para la población de Carhuamayo, demostró que no hubo diferencia significativa entre ambos grupos, (p=0,243), (Figura 2).

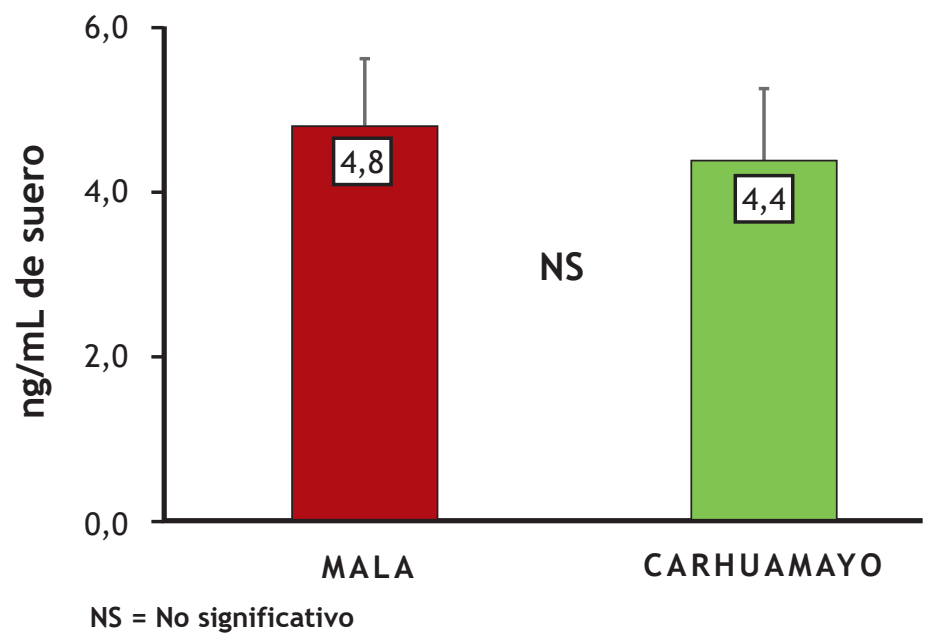

Figura 2. Niveles medios de $\mathrm{sCD} 36$ ( $\mathrm{ng} / \mathrm{mL}$ de suero)

Cuando se analizaron los resultados de SCD36 según género, se encontró que el promedio sérico en la totalidad de las mujeres $(n=60)$ y de los hombres $(n=25)$ no presentaban diferencia estadística: 4,6 $\pm 1,7$ vs 4,7 $\pm 1,1$ ( $p=0,793)$. Se encontró diferencia estadística significativa entre los valores medios séricos de sCD36 según IMC (Figura 3).

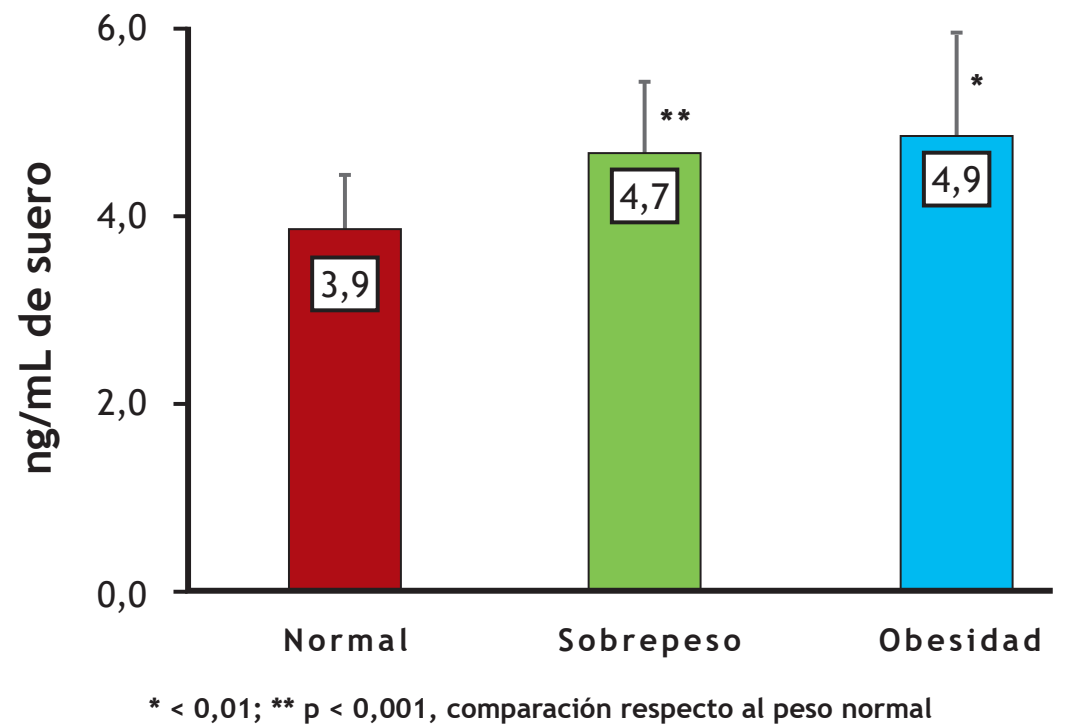

Figura 3. Niveles medios de $\mathrm{sCD} 36(\mathrm{ng} / \mathrm{mL}$ de suero) según IMC

También se evaluó el grado de asociación entre el nivel de sCD36 sérico y las demás variables observadas (Tabla 4). Se encontró una relación directa entre niveles séricos de sCD36 y peso e IMC ( $(p=0,016$ y 0,002 , respectivamente). No se encontró asociación significativa entre el nivel sérico de este metabolito con los demás parámetros evaluados. 
Tabla 4. Correlación entre nivel de SCD36 sérica y variables de riesgo cardiometabólico

\begin{tabular}{|c|c|c|}
\hline Variable & $\mathbf{r}$ & $\mathbf{p}$ \\
\hline Edad, años & 0,033 & 0,393 \\
\hline Peso, $\mathbf{k g}$ & 0,261 & $0,016^{*}$ \\
\hline Talla, $\mathbf{m}$ & 0,137 & 0,211 \\
\hline IMC, $\mathbf{k g} / \mathbf{m} \mathbf{2}$ & 0,331 & $0,002^{*}$ \\
\hline Cintura, $\mathbf{c m}$ & 0,093 & 0,410 \\
\hline Presión sistólica, $\mathbf{m m H g}$ & 0,227 & 0,069 \\
\hline Presión diastólica, $\mathbf{m m H g}$ & 0,198 & 0,071 \\
\hline Glucosa, $\mathbf{m g} / \mathbf{d L}$ & 0,087 & 0,428 \\
\hline Colesterol, $\mathbf{m g} / \mathbf{d L}$ & 0,04 & 0,717 \\
\hline HDL-colesterol, $\mathbf{m g} / \mathbf{d L}$ & $-0,094$ & 0,394 \\
\hline LDL-colesterol, $\mathbf{m g} / \mathbf{d L}$ & $-0,024$ & 0,830 \\
\hline Triglicéridos, $\mathbf{m g} / \mathbf{d L}$ & 0,179 & 0,102 \\
\hline Hemoglobina $(\mathbf{g} / \mathbf{d L})$ & $-0,048$ & 0,662 \\
\hline
\end{tabular}

\section{* Estadísticamente significativo ( $r$ de Pearson)}

\section{DISCUSIÓN}

Se realizó un estudio transversal en una muestra de sujetos oriundos de las grandes alturas (Carhuamayo, $4100 \mathrm{msnm}$ ) y en otra del nivel del mar (Mala, $30 \mathrm{~m}$ ) para estimar la asociación entre los niveles de sCD36 sérico y algunas variables consideradas como factores de riesgo de enfermedades cardiometabólicas. Así, se encontró que la población de Mala en promedio, presentó mayor peso e IMC que la de la altura; en cambio, la presión arterial diastólica y sistólica resultaron similares en ambos grupos y estaban dentro de los límites establecidos como normales ${ }^{(26)}$.

Los niveles de $\mathrm{Hb}$ fueron mayores en el grupo de altura que en el grupo del nivel del mar; el incremento de $\mathrm{Hb}$ en residentes de la grandes alturas es uno de los principales mecanismos de adaptación a la hipoxia de estas altitudes, junto con el incremento en el número de hematíes, y en el hematocrito ${ }^{(25)}$.

Los niveles de glucemia del grupo de altura fueron significativamente menores que los del grupo de nivel del mar, lo que confirma una vez más que en la altura normalmente los valores medios de glucosa sérica son menores que sus similares del nivel $\operatorname{mar}^{(25)}$.

Los resultados de perfil lipídico, obtenidos en este estudio, indican que los valores séricos de colesterol total, HDL-col y LDL-col estaban dentro del rango considerado deseable según la literatura ${ }^{(26)}$ para la población de Carhuamayo y eran similares a los encontrados por otros investigadores $(27,28)$. En el caso de los habitantes de la ciudad de Mala solamente el colesterol total se encontró dentro de los valores considerados como "riesgo moderado". Aunque los niveles séricos de TG aquí observados también están dentro de límites considerados como "riesgo moderado", difieren de los referidos por otros autores, quienes encontraron valores altos de TG en otras poblaciones andinas ${ }^{(27,28)}$. Se ha identificado una forma soluble de CD36 en el plasma humano, y se ha encontrado niveles altos de esta sustancia en pacientes con diabetes tipo 2 y en obesos, por lo cual se le propone como un marcador del síndrome metabólico $\mathrm{y}$ un potencial marcador de la aterosclerosis ${ }^{(1,5)}$.

El comportamiento estadístico de los datos de sCD36 séricos, según el histograma obtenido, muestra que la población estudiada sigue una distribución bimodal, similar a la observada por Handberg et al ${ }^{(14)}$, quienes estudiaron una muestra mayor de más 1000 sujetos, en tanto que el presente estudio comprendió solamente 85 .

Los resultados del presente trabajo, muestran que, los valores medios de sCD36 en el grupo de Mala fueron similares a los de Carhuamayo; lo que significa que el nivel sérico de este metabolito es independiente de la altitud. De otro lado, en este estudio, se observó que sCD36 fue significativamente mayor en los sujetos con sobrepeso y obesidad, respecto de los sujetos con peso normal $(4,7 \mathrm{ng} / \mathrm{mL}, 4,9 \mathrm{ng} / \mathrm{mL}$ y $3,9 \mathrm{ng} / \mathrm{mL})$ respectivamente, estos resultados son similares a estudios anteriores ${ }^{(12,16)}$. Esto se debe posiblemente al incremento del número de plaquetas y monocitos en los sujetos obesos, y la forma circulante del receptor CD36 se asocia principalmente a las plaquetas, leucocitos y las células endoteliales, pues se ha demostrado una relación entre obesidad e inflamación. También observó una correlación positiva altamente significativa de sCD36 con el peso e IMC en la población total. Resultados similares han sido presentados en otros estudios $^{(12,16)}$ y confirmarían un incremento de la expresión del receptor CD36 en el tejido adiposo ${ }^{(21)}$.

Nuestra investigación tiene algunas limitaciones. El tamaño muestral que logramos colectar no nos permite estudiar la asociación entre los niveles de sCD36 y la prevalencia de síndrome metabólico, y el hecho de que nuestro estudio comprende personas aparentemente sanas sin enfermedades ateroscleróticas, ni diabéticas; sólo hemos podido estudiar las asociaciones entre factores de riesgo cardiovascular que están dentro de límites normales 
con los niveles de sCD36. Es necesario aumentar el número de sujetos en ambas poblaciones para dilucidar el papel que representaría este receptor en poblaciones que viven en las grandes alturas.

En conclusión, en el presente estudio se encontró que no hay diferencia significativa en los promedios de los niveles séricos de sCD36 entre las poblaciones de Carhuamayo (4160 $4100 \mathrm{msnm}$ ) y Mala (30 msnm) y que los niveles de sCD36 se asocian con incremento de tejido adiposo, por lo que puede ser considerado como marcador potencial temprano de síndrome metabólico en ambas poblaciones.

\section{REFERENCIAS BIBLIOGRÁFICAS}

1. Febbraio M, Hajjar D, Silverstein R. CD36: a class B scavenger receptor involved in angiogenesis, atherosclerosis, inflammation, and lipid metabolism. J Clin Invest. 2001;108(6):785-90

2. Silverstein R. Inflammation, atherosclerosis, and arterial thrombosis: role of the scavenger receptor CD36. Cleve Clin J Med. 2009; 76 (Suppl 2): S27-S30.

3. Hoosdally S, Andress E, Wooding C, Marin C, Linton K. The human scavenger receptor $\mathrm{CD} 36$; glycosylation status and its role in trafficking and function. J Biol Chem. 2009; 284(24):16277-88.

4. Abumrad N, El-Maghrabi M, Amri E, Lopez E, Grimaldi P. Cloning of a rat adipocyte membrane protein implicated in binding or transport of long chain fatty acids that is induced during preadipocyte differentiation. Homology with human CD36. J Biol Chem.1993; 268(24): 17665-8.

5. Thorne R, Mhaidat N, Ralston K, Burns G. CD36 is a receptor for oxidized high density lipoprotein: implications for the development of atherosclerosis. FEBS Lett. 2007; 581(6):1227-32

6. Susztak K, Ciccone E, McCue P, Sharma K, Bottinger E. Multiple metabolic hits converge on $\mathrm{CD} 36$ as novel mediator of tubular epithelial apoptosis in diabetic nephropathy. PLoS Med. 2005; 2(2): e45.

7. Libby P. Inflammation in atherosclerosis. Nature. 2002; 420: 868-74.

8. Endeman G, Stanton L, Madden K, Bryant C, White R, Protter A. CD36 is a receptor for oxidized low density lipoprotein. J Biol Chem. 1993; 268(16): 11811-6

9. Podrez E, Schmitt D, Hoff H, Hazen S. Myeloperoxidase generated reactive nitrogen species convert $L D L$ into an atherogenic form in vitro. J Clin Invest. 1999; 103(11):1547-60

10. Jiang $Y$, Wang $M$, Huang $K$, Zhang Z, Shao N, Zhang $Y$, et al. Oxidized low density lipoprotein induces secretion of interleukin $1 \mathrm{~b}$ by macrophages via reactive oxygen species dependent NLRP3 inflammasome activation. Biochem Biophys Res Commun. 2012; 425(2):121-6.

11. Handberg A, Levin $\mathrm{K}$, Hojlund $\mathrm{K}$, Beck-Nielsen $\mathrm{H}$. Identification of the oxidized low density lipoprotein scavenger receptor CD36 in plasma, a novel marker for insulin resistance. Circulation. 2006; 114(11):1169-76.

12. Fernandez-Real JM, Handberg A, Ortega F, Hojlund K, Vendrell J, Ricart W. Circulating soluble CD36 is a novel marker of liver injury in subjects with altered glucose tolerance. J Nutr Biochem. 2009; 20(6): 477-84.

13. Ramos-Arellano L, Muñoz-Valle J, De la Cruz-Mosso U, Salgado-Bernabe A, Castro-Alarcon N, Parra-Rojas I. Circulating CD36 and ox-LDL levels are associated with cardiovascular risk factors in young subjects. BMC Cardiovasc Disord. 2014; 14(1): 54

14. Handberg K, Hojlund A, Gastaldelli A, Flyvbjerg A, Dekker J, Petrie J, et al. Plasma SCD36 is associated with markers of atherosclerosis, insulin resistance and fatty liver in a nondiabetic healthy population. J Intern Med. 2012, 271(3): 294-304

15. Handberg A, Skjelland M, Michelsen A, Sagen E, Krohg-Sorensen K, Russell $D$, et al. Soluble CD36 in plasma is increased in patients with symptomatic atherosclerotic carotid plaques and is related to plaque instability. Stroke. 2008; 39(11): 3092-5.

16. Park Y. Cd36, a scavenger receptor implicated in atherosclerosis. Experimental \& molecular medicine. 2014;46(6):e99

17. Lam M, Tan K, Lam K. Glycoxidized lowdensity lipoprotein regulates the expression of scavenger receptors in THP1 macrophages. Atherosclerosis. 2004; 177(2): 313-20
18. Younis N, Soran H, Sharma R, Charlton-Menys V, Durrington P. Lipoprotein glycation and atherogenesis. Clin Lipidol. 2009; 4(6):781-90

19. Sampson M, Davies I, Braschi S, Ivory K, Hughes D. Increased expression of a scavenger receptor (CD36) in monocytes from subjects with type 2 diabetes. Atherosclerosis. 2003; 167(1): 129-34

20. Furuhashi $M$, Ura N, Nakata T, Shimamoto K. Insulin sensitivity and lipid metabolism in human CD36 deficiency. Diabetes Care. 2003; 26(2): 471-4

21. Griffin E, Re A, Hamel N, Fu C, Bush H, McCaffrey T, et al. A link between diabetes and atherosclerosis: glucose regulates expression of CD36 at the level of translation. Nat Med. 2001; 7(7): 840-846

22. Bonen A, Tandon N, Glatz J, Luiken J, Heigenhauser G. The fatty acid transporter FAT/CD36 is upregulated in subcutaneous and visceral adipose tissues in human obesity and type 2 diabetes. Int J Obes. 2006; 30(6): 877-83

23. Liang C, Han S, Okamoto H, Carnemolla R, Tabas I, Accili D, Tall A. Increased CD36 protein as a response to defective insulin signaling in macrophages. J Clin Invest. 2004; 113(5): 764-73

24. Segura-Vega L, Regulo-Agusti C, Ramírez J. Factores de Riesgo de las Enfermedades Cardiovasculares en el Perú. Estudio TORNASOL. Rev peru cardiol. 2006; 32:82-128

25. West J, Schoene R, Luks A, Milledge J. High Altitude Medicine and Physiology. 5nd ed. CRC Press, Taylor \& Francis Group Boca Raton; 2013

26. National Cholesterol Education Program (NCEP). Third Report of the National CholesterolEducation Program (NCEP) Expert Panel on Detection, Evaluation, and Treatment of HighBlood Cholesterol In Adults (Adult Treatment Panel III) final report. Circulation. 2002;106(25):3143-421

27. Mohanna S, Baracco R, Seclen S. Lipid profile, waist circumference, and body mass index in a high altitude population. High Alt Med Biol. 2006; 7(3):245-55.

28. Gonzáles G, Tapía V. Asociación de los diferentes niveles de hipoxemia en la altura con el perfil lipídico y la glucemia en varones y mujeres a 4,100 m de altitud en los Andes Centrales del Perú. Endocrinol Nutr. 2012; 60(2):79-86.

\section{Fuentes de financiamiento:}

Este artículo ha sido financiado por los autores.

\section{Conflictos de interés:}

Los autores declaran no tener ningún conflicto de interés.

\section{Correspondencia:}

Elizabeth Carranza Alva

Dirección: Av. Alfonso Ugarte 880. Lima 1, Perú.

Instituto Nacional de Biología Andina, Facultad de Medicina, UNMSM.

Teléfono: 997197163

Correo electrónico: ecarranzalva@gmail.com

Recibido: 11 de agosto de 2017

Evaluado: 17 de agosto de 2017 Aprobado: 31 de agosto de 2017

( $)$ La revista. Publicado por Universidad de San Martín de Porres, Perú. (cc) Br Licencia de Creative Commons Artículo en acceso abierto bajo términos de Licencia Creative Commons Atribución 4.0 Internacional. (http://creativecommons.org/licenses/by/4.0/) 